and the results of large observational studies such as SITS-MOST and concluded that tPA was effective in the treatment of acute ischaemic stroke. ${ }^{3}$ Another pooled analysis of individual data of six tPA trials showed that, even within a 3-hour window, earlier treatment results in a better outcome $(0-90$ minutes: odds ratio (OR) 2.11, 95\% confidence interval (CI) 1.33 - 3.55; 90 - 180 minutes: OR 1.69; 95\% CI 1.09 - 2.62) and suggested a benefit up to 4.5 hours. ${ }^{4}$ The ECASS III trial found tPA to be effective when provided up to 4.5 hours after stroke onset (OR 1.34, 95\% CI $1.02-1.76, p=0.04$ ). There was a significant increase in symptomatic intracranial haemorrhage $(2.7 \%$ v. $0.3 \%)$, but no significant effect on mortality. In another study the SITS investigators compared 664 patients with ischaemic stroke treated between 3 and 4.5 hours with 11865 patients treated within 3 hours. ${ }^{5}$ There were no significant differences between the 3 - 4.5-hour cohort and the 3-hour cohort for any outcome measures, confirming that tPA remains safe when given between 3 and 4.5 hours after the onset of symptoms in ischaemic stroke patients who otherwise fulfil the European summary of product characteristics criteria. A systematic review of four trials, including ECAS III, confirmed that TPA given between 3 and 4.5 hours after stroke onset is associated with an increased chance of favourable outcome (OR $1.31,95 \%$ CI $1.10-1.56, p=0.002)$ with no significant difference in mortality compared with placebo, thus strengthening the evidence that treatment with IPA in the 3 - 4.5-hour window is beneficial and should therefore be considered for stroke patients who present during this time window. ${ }^{6}$

The recommendation for the use of IVI tPA within 4.5 hours of onset of ischaemic stroke in the new South African guideline is the same as in the guidelines of other countries including the USA, Canada, the European Union, Scandinavia and Australia. The evidence shows that IVI tPA treatment benefits selected patients but should be delivered in well-equipped and skilled emergency units and/or stroke units with adequate expertise and infrastructure for monitoring, rapid assessment and investigation of acute patients. Such patients should be offered treatment with tPA provided the potential risks and benefits are explained to them and their attendant families. Collaboration is recommended between clinicians in pre-hospital services, emergency medicine, neurology and neuroradiology for prompt identification of potentially eligible patients, patient selection, audit and quality improvement initiatives.

\footnotetext{
1. Wardlaw JM, Murray V, Berge E, del Zoppo G. Thrombolysis for acute ischaemic stroke. Stroke 2010;41:e445-e446.

Wahlgren N, Ahmed N, Da'valos A, et al.; SITS-MOST Investigators. Thrombolysis with alteplase for 2. Wahlgren N, Ahmed N, Da valos A, et al.; SITS-MOST Investigators. Thrombolysis with alteplase for
acute ischaemic stroke in the Safe Implementation of Thrombolysis in Stroke-Monitoring Study (SITSacute ischaemic stroke in the Safe Implementation of Thrombolysis in Stroke-Monitoring Study (SITS-
MOST): an observational study. Lancet 2007;369:275-282. [published correction appears in Lancet MOST): an observational study. Lancet 2007;369:275-282. [published correction appears in Lance 2007;369:826

3. National Institute for Health and Clinical Excellence. Altepase for the treatment of acute ischaemic stroke (TA 122). London: NICE, 2007; updated 2010.

Hacke W, Donnan G, Fieschi C, et al. Association of outcome with early stroke treatment: pooled analysis of ATLANTIS, ECASS, and NINDS rt-PA stroke trials. Lancet 2004;363:768-774.

Wahlgren N, Ahmed N, Dávalos A, et al. Thrombolysis with alteplase 3-4.5 h after acute ischaemic stroke (SITS-ISTR): an observational study. Lancet 2008;372:1303-1309.

Lansberg MG, Bluhmki E, Thijs VN. Efficacy and safety of tissue plasminogen activator 3 to 4.5 hours after acute ischemic stroke. A meta-analysis. Stroke 2009;40:2438-2441.
}

\section{Will National Health Insurance ensure the national health?}

To the Editor: The good intentions in the proposed National Health Insurance (NHI) scheme are not doubted, but I suspect that the authorities have not been correctly advised on why the health system is often inadequate.

South Africa's health system provides private health care access to some, but the large majority depends on the state for health services. Private health care appears to offer a better service than state health care because of a lack of adequate state sector infrastructure. However, injecting massive NHI funding into state health will result in a burgeoning bureaucracy and little to improve our citizens' health experience.

Infrastructure, from equipment to health care personnel, is limited in the state health system and patients experience many irritations and inconveniences because of the way it operates.

There is no reason why repeat outpatients cannot have appointments instead of all having to attend the health facility before dawn only to be seen hours later. With appointments the number of patients in a health facility would become more manageable and patients who work would not lose pay by waiting unnecessarily.

Why do patients have to return, often after long journeys, to collect repeat medication instead of accessing it at their nearest state health facility? Private sector prescriptions are on a computer and available at supermarket pharmacies nationwide. State health facilities can use such computer programmes and reduce outpatient and clinic numbers by $30 \%$. And why do supermarkets have better baby-changing facilities than our health facilities, which often do not have any at all?

Patient self-triage is another serious patient-perceived problem. For budgetary and administrative reasons state health services are classified as primary or district, secondary or regional, and tertiary or academic. The family with a sick baby is expected to self-triage the baby into one of these categories. Heaven help them if they appear at a secondary or tertiary hospital with a primary care condition, as they may be denied treatment and referred back. Since when do health facilities turn away patients? Health facilities must recognise that their concept of an emergency differs from that of a lay person and act accordingly!

The state health services have some excellent health practitioners, but there are not enough of them. Most are concentrated in urban areas and medical schools, and although this is unlikely to change, the rural paucity of practitioners could be addressed in part by the following.

Firstly, address the overall numbers of health practitioners in the state services. One cannot buy, but must attract and retain the best personnel. This is not just about money, and the nine provincial health departments and the eight university medical schools must play a role in this joint approach and responsibility. Provinces with medical schools must accept the obligation of providing state-of-theart equipment and consumables to the teaching environment. It is unacceptable that cardiac procedures be delayed because the budget for stents is insufficient, or that patients should suffer long delays because the prosthetic hips budget is exhausted.

Secondly, as part of their co-responsibility for patients the medical schools must recognise that the health department's responsibility for the patients in a teaching hospital extends throughout the province. In recognising this health and teaching continuum the medical schools should co-operate in outreach programmes throughout and across provinces to take treatment to where the patients live, subject to the provinces meeting their equipment obligations. They must also recognise the medical practitioner needs in South Africa and support the front-line health care disciplines of family medicine and emergency medicine.

The present state health system could be remedied by wise investment in real patient care at less than an NHI would cost, and without creating another bureaucracy.

\section{Alan Mac Mahon}

alanjean@telkomsa.net 